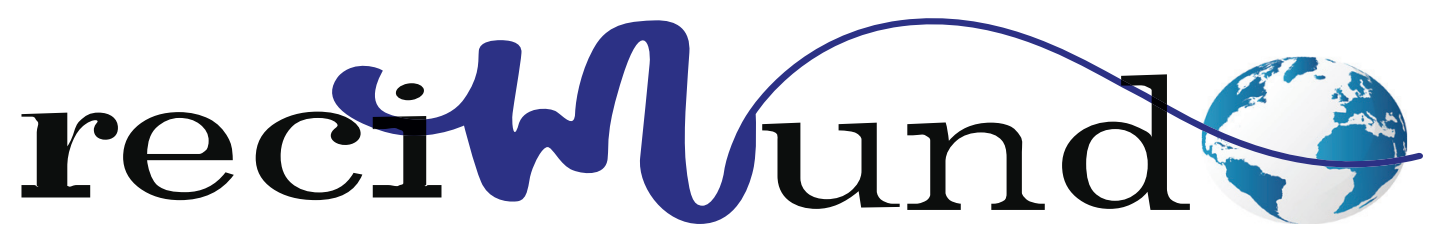

Revista Científica Mundo de la Investigación y el Conocimiento

DOI: 10.26820/recimundo/4.(4).noviembre.2020.300-308

URL: http://recimundo.com/index.php/es/article/view/953

EDITORIAL: Saberes del Conocimiento

REVISTA: RECIMUNDO

ISSN: 2588-073X

TIPO DE INVESTIGACIÓN: Artículo de revisión

CÓDIGO UNESCO: 1203.04 Inteligencia Artificial; 1203.05 Sistemas Automatizados de Producción

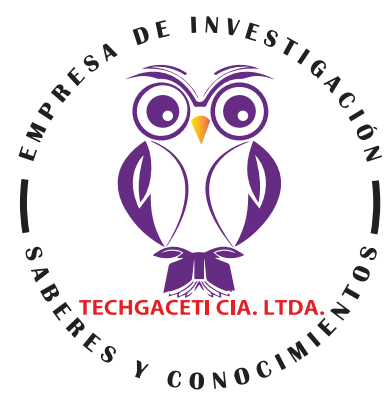

PAGINAS: $300-308$

\title{
Gemelos digitales y su evolución en la industria
}

Digital twins and their evolution in the industry

Gêmeos digitais e sua evolução na indústria

Michelle Varas Chiquitoํㅜ Juan Carlos García Plua²; Mariana Bustamante Chong ${ }^{3}$; César Bustamante Chong ${ }^{4}$

RECIBIDO: 10/07/2020 ACEPTADO: 02/09/2020 PUBLICADO: 10/11/2020

1. Magíster en Diseño Curricular; Ingeniera en Ciencias Computacionales; Docente Investigadora tiempo completo de la Universidad de Guayaquil, Ecuador; michelle.varasch@ug.edu.ec; iD https://orcid.org/0000-0001-6166-5303

2. Magíster en sistemas de Información Gerencial Licenciado en sistemas de información; Docente Investigador tiempo completo de la Universidad de Guayaquil; Ecuador; juan.garciap1@ug.edu.ec; iD https://orcid.org/0000-0002-3377-3216

3. Doctorando en Administración de Empresas Pontificia Universidad católica Argentina Santa María de los Buenos Aires (Argentina); Magíster en Administración Pública mención Desarrollo Institucional; Economista; Docente Investigadora tiempo completo de la Universidad Estatal del sur de Manabí; Ecuador; mariana.bustamante@unesum.edu.ec; (DD https://orcid. org/0000-0002-5815-3267

4. Doctorando en Administración de Empresas Pontificia Universidad católica Argentina Santa María de los Buenos Aires (Argentina); Magíster en Administración de Empresas mención en Mercadotecnia; Ingeniero en Ciencias Computacionales. Docente investigador tiempo completo de la Universidad Tecnológica Ecotec; Ecuador; cbustamante@ecotec.edu.ec; (iD https://orcid.org/0000-0003-0150-1088

CORRESPONDENCIA

Michelle Varas Chiquito

michelle.varasch@ug.edu.ec

Guayaquil, Ecuador 


\section{RESUMEN}

Los gemelos digitales hacen referencia a una tecnología de software con el que se trata de representar digitalmente una realidad física, bien sea esta un producto, proceso o sistema; misma que a la par se apoya en otros recursos tecnológicos tales como: Big Data, IoT, Inteligencia Artificial, Cloud Computing y Machine Learning, entre otras, con la finalidad de proporcionar herramientas para la toma de decisiones, implementando adicionalmente capacidades de simulación y predicción. El objeto del presente trabajo investigativo consiste en efectuar una sucinta revisión de la bibliografía científico académica disponible en mediante el uso de la internet, y a su vez, de determinadas bases de datos y buscadores especializados, a fines de describir y exponer sobre los gemelos digitales (GD) y su evolución en la industria, razón por la que se estima desarrollar algunos conceptos básicos sobre los GD, su origen, evolución, utilidades (ventajas) y las barreras o limitaciones tecnológicas para su aplicación. En las conclusiones fue posible deducir que, a pesar de que la tecnología de los GD representa un avance relativamente reciente, los expertos aseguran que ha tenido una rápida evolución y aplicación en varios tipos de industria; que la finalidad de crear un GD no es la conseguir la consumación de la entidad o réplica virtual en sí misma, sino más bien los beneficios que de éste es posible obtener; y que la evolución de los GD estará estrechamente vinculada a la propia evolución tecnológica y a la, cada vez mayor, admisión en distintas áreas e infraestructuras digitales en industrias, productos, ciudades y otras.

Palabras clave: Realidad Virtual, Big Data, Inteligencia Artificial, Cloud Computing, Machine Learning.

\section{ABSTRACT}

Digital twins refer to a software technology that tries to digitally represent a physical reality, be it a product, process or system; At the same time, it is supported by other technological resources such as: Big Data, IoT, Artificial Intelligence, Cloud Computing and Machine Learning, among others, in order to provide tools for decision-making, additionally implementing simulation and prediction capabilities. . The purpose of this research work is to carry out a succinct review of the scientific-academic bibliography available through the use of the Internet, and in turn, of certain databases and specialized search engines, in order to describe and expose on digital twins (GD ) and its evolution in the industry, which is why it is estimated to develop some basic concepts about DG, its origin, evolution, utilities (advantages) and technological barriers or limitations for its application. In the conclusions it was possible to deduce that, despite the fact that DG technology represents a relatively recent advance, experts assure that it has had a rapid evolution and application in various types of industry; that the purpose of creating a GD is not to achieve the consummation of the entity or virtual replica itself, but rather the benefits that can be obtained from it; and that the evolution of the DG will be closely linked to the technological evolution itself and to the increasing admission in different areas and digital infrastructures in industries, products, cities and others.

Keywords: Virtual Reality, Big Data, Artificial Intelligence, Cloud Computing, Machine Learning.

\section{RESUMO}

Os gêmeos digitais referem-se a uma tecnologia de software que tenta representar digitalmente uma realidade física, seja um produto, processo ou sistema; Ao mesmo tempo, conta com o suporte de outros recursos tecnológicos como: Big Data, IoT, Inteligência Artificial, Cloud Computing e Machine Learning, entre outros, de forma a fornecer ferramentas para a tomada de decisão, implementando adicionalmente capacidades de simulação e previsão. . O objetivo deste trabalho de pesquisa é realizar uma revisão sucinta da bibliografia científico-acadêmica disponível através do uso da Internet e, por sua vez, de algumas bases de dados e buscadores especializados, com o objetivo de descrever e expor sobre gêmeos digitais (GD ) e sua evolução na indústria, pelo que se estima desenvolver alguns conceitos básicos sobre GD, sua origem, evolução, utilidades (vantagens) e barreiras ou limitações tecnológicas para a sua aplicação. Nas conclusões foi possível deduzir que, apesar de a tecnologia de GD representar um avanço relativamente recente, os especialistas asseguram que ela teve uma evolução rápida e aplicação em vários tipos de indústria; que o propósito de criar um GD não é atingir a consumação da entidade ou réplica virtual em si, mas sim os benefícios que podem ser obtidos a partir dela; e que a evolução da DG estará intimamente ligada à própria evolução tecnológica e à crescente admissão em diferentes áreas e infraestruturas digitais em indústrias, produtos, cidades e outros.

Palavras-chave: Realidade Virtual, Big Data, Inteligência Artificial, Cloud Computing, Machine Learning. 


\section{Introducción}

Un gemelo digital (GD), traducción derivada de la expresión en inglés: Digital Twin (DT); consiste en la creación de una réplica virtual, a imagen y semejanza de un producto, proceso o sistema tangible, al cual se le vinculan datos en tiempo real que pueden ser captados mediante sensores o de otros recursos tecnológicos asociados con el Big Data. Luego de recabar toda esa información se procesa con Inteligencia Artificial, Cloud Computing (computación en la nube) y Machine Learning (aprendizaje automático) para así generar la representación viva que puede sentir, pensar y actuar. (Rodríguez, 2019; Iberdrola, 2020)

La Cámara Oficial de Comercio, Industria y Navegación de Valencia (Cámara Valencia) de España señala que "...los gemelos digitales vigilan el ciclo de vida de un proceso replicando su funcionamiento en un modelo virtual que sirve como base para la experimentación." (Cámara Valencia, s.f.)

Según Ayala (citado en Mapfre Global Risks, 2020), en la investigación efectuada por la consultora Gartner se indica que cerca del $50 \%$ de las industrias usará gemelos digitales para el 2021, acción que favorecerá a nivel global el crecimiento de la eficiencia en un $10 \%$.

Se estima que el mercado de gemelos digitales crecerá de los 3.800 millones de dólares de 2019 hasta los 35.800 millones en 2025, con una tasa compuesta anual del $45,4 \%$, según la consultora Markets and Markets. Los factores que impulsan la demanda de gemelos digitales incluyen la creciente adopción de tecnologías emergentes como IoT y la nube -para su implementación- y la prometedora perspectiva de su incorporación a industrias como la aeroespacial, defensa, automotriz, transporte y atención médica. (Mapfre Global Risks, 2020)
El objeto del presente trabajo investigativo consiste en efectuar una sucinta revisión de la bibliografía cientificoacadémica disponible en mediante el uso de la internet, y a su vez, de determinadas bases de datos y buscadores especializados, a fines de describir y exponer sobre los gemelos digitales (GD) y su evolución en la industria, razón por la que se estima desarrollar algunos conceptos básicos sobre los GD, su origen, evolución, utilidades (ventajas) y las barreras o limitaciones tecnológicas para su aplicación. A continuación, se explica la metodología investigativa, y en la siguiente sección se deja en evidencia los resultados obtenidos de la revisión bibliográfica, exponiendo y describiendo sobre los aspectos ya mencionados en base a las fuentes encontradas y al final se presentan las conclusiones correspondientes,

\section{Materiales y Métodos}

Basando este estudio en un diseño de investigación bibliográfico y en una metodología de revisión, se procedió a efectuar búsquedas de fuentes de información con carácter cientificoacadémico, mediante el uso de buscadores especializados (Refseek, Google Académico) y algunas bases de datos reconocidas como: Scopus, Dialne y, Base.

Con el uso de palabras claves y algunos operadores lógicos, se constituyen los siguientes descriptores de búsqueda "Gemelos digitales"; Gemelos digitales|Digital Twins +evolución +industria; "Digital Twins AND evolution", entre otras configuraciones y adaptaciones. A los resultados obtenidos con éstos, se les aplicaron dos filtros principales, que fueron: Idioma (Español e Inglés) y Periodo de publicación (2011 - 2020); no obstante, adicionalmente se emplearon otras factores de refinamiento disponibles en las otras plataformas de búsqueda consultadas, entre ellas: tipo de material bibliográfico (artículos originales - papers, libros, secciones de libros, ebooks, tesis de 
grado, postgrado o doctorado, publicaciones empresariales o de instituciones formales $u$ oficiales, notas o noticias periodísticas, boletines informativos, conferencias, y otros), área de investigación (ciencias informáticas, ingeniería, tecnología y similares), acceso (completo y, abierto o cerrado) entre otros.

Los resultados de las búsquedas (referidos a los enlaces de direcciones web que contenían cada uno de los distintos tipos de recursos bibliográficos) representaron en: Google Académico =778; Refseek =10100, Scopus $=4520$, Dialnet $=58$ y en Base $=5$, pero, no todos estos recursos fueron inspeccionados y en base al objetivo planteado, se consideró resisar solo hasta los primeros 200 títulos de cada uno de los medios de búsqueda utilizados, priorizando aquellos en los que se encontrara la mayor relación posible entre el título de la otra mostrado y el que se ha definido en este estudio, y de éstos, sólo aquellos en los que se pudiera evidenciar mediante una breve lectura de sus resúmenes y/o contenidos, que expusieran lo relativo a conceptos y definiciones básicas, origen, evolución, barreras o limitaciones y utilidades (ventajas) de los GD.

Se descartó todo el material que se halló repetido, es decir, aquellas publicaciones que, siendo una sola, se encontraba alojada en bases de datos o repositorios distintos. Finalmente, alcanzaron 12 publicaciones para sustentar las ideas con las que se alcanzó el objetivo propuesto.

\section{Resultados}

\section{Origen de los GD}

Ha de tenerse claro que, el GD no se trata de una idea nueva pues su concepto tuvo origen en el transcurso de la penúltima década del siglo pasado, en base a los trabajos realizados por la Agencia Espacial de los Estados Unidos de América (NASA, por siglas en inglés), al efectuar simulacio- nes sobre el comportamiento de las naves o equipamientos para asegurar la viabilidad de ciertas misiones y velar por la integridad física de los astronautas. Lo que en la actualidad ha favorecido la expansión y desarrollo de los GD hacia otros sectores y diferentes tipos de organizaciones han sido los avances y mayor accesibilidad a las otras tecnologías en las que se apoya y beneficia la tecnología del GD, tales como el Big Data, Cloud Computing o el internet de las cosas (IoT), y también de la reducción de costes en los sensores necesarios para el método de GD. (Alfonso, 2018; BBVA, 2019)

Ahora bien, fueron Michael Grieves junto a John Vickers los expertos que hablaron en 2003, durante una participación conjunta en la Universidad de Michigan, sobre la viabilidad en la creación de representaciones digitales de sistemas físicos que tuvieran entidad por sí mismas. Ya en 2018 pasado la consultora Gartner identificó esta tecnología como una de las diez tendencias de ese año. (Iberdrola, 2020) Entonces, ese último dato bien puede asumirse como una notable evidencia de la rápida evolución de dicha tecnología.

Los gemelos digitales son soluciones que buscan representar digitalmente una realidad física, generando un conocimiento que pivota alrededor de los datos, con objetivo de proporcionar herramientas para la toma de decisiones, implementando adicionalmente capacidades de simulación y predicción. Y la diferencia de estas soluciones respecto otras es precisamente su clara búsqueda de reproducir, procesar, simular, predecir lo que puede ocurrir en el mundo físico.

En este nivel de dar respuesta a problemas, otros sistemas como ERP, MES, SCADA, cuadros de mando, etc. tratan de avanzar en estas soluciones.

Podemos también decir que un Gemelo Digital es un software que construye una so-

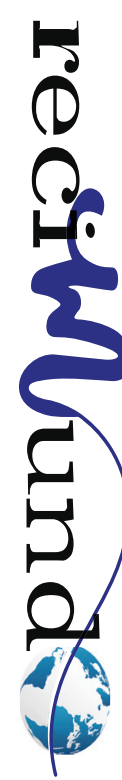


lución, integrando información y procesos entre otros softwares y aplicando tecnología para conseguir operar con el mundo físico, simular, predecir y presentar al usuario lo que necesita de una forma intuitiva para que pueda tomar decisiones más rápidas y fiables. (De Juana, 2020)

Por su parte, Leclère (2018) define al GD como la maquetación virtual de un producto, proceso o servicio.

Es un puente entre el mundo virtual y el mundo físico que permite simular escenarios de funcionamiento desde la concepción a través de todo el ciclo de vida del producto, analizar datos para mejorar su funcionamiento y realizar monitorizaciones para identificar problemas antes de que ocurran. (pág. 30)

Se considera hasta aquí entonces aclarar que, en base a lo referido al inicio de esta entrega y a la idea antes citada que, en concreto, según el uso al que pudieran estar dirigidos, son tres los tipos de GD que existen: de producto, de producción y de rendimiento. (Geinfor, s.f.; Mapfre Global Risks, 2020)

Un requisito para la construcción de un Gemelo Digital es utilizar todos los datos que se encuentren disponibles. Sin embargo, la gran cantidad de datos que genera una planta en continuo supone un reto en términos tanto de almacenamiento como de procesamiento. Este hecho requiere la aplicación de tecnologías Big Data Analytics, para gestionar eficientemente los datos en tiempo real, combinados junto con la información histórica y de contexto (logística, ventas, almacén, entorno). Por otra parte, para la creación del modelo predictivo que fundamenta un Gemelo Digital, es necesario la aplicación de técnicas punteras en el ámbito del Machine Learning. Sólo mediante la introducción de ambas facetas, es posible crear un Gemelo Digital que proporcione simulaciones precisas a partir tanto de su operativa actual como de estados pasados y futuros. (ITI, 2020)

\section{Evolución de los GD}

Saracco (2019), señala que los GD han evolucionado lo largo de los años, y seguirán evolucionando en la próxima década. Se están volviendo en un componente clave de la transformación digital, y a veces son un facilitador de la transformación digital. Además, explico que:

Los modelos digitales se basan en CAD, y es fácil entender por qué varias industrias los han adoptado. Las empresas de suministro y fabricación emplean procesos digitales y máquinas controladas digitalmente para crear hilos digitales; los productos están diseñados para conectarse a través de una red de comunicación y retransmitir datos operativos utilizados para producir sombras digitales. Los gemelos digitales contribuyen a la cadena de valor, y las industrias, incluyendo la arquitectura, la ingeniería, la construcción y el cuidado de la salud están empezando a considerar la creación y explotación de ellos. (pág. 59)

Éste experto asegura que cada vez es mayor el consenso respecto a que, en el transcurso de los próximos 10 años, las aplicaciones de gemelos digitales se expandirán en muchas áreas y las capacidades de los modelos mejorarán. Una muestra de ello se evidencia en áreas de la salud; siendo válido el desarrollo de empresas como General Electric, quien ha comenzado a utilizar gemelos digitales para simular y monitorear las operaciones hospitalarias; o también es válida la incursión de ésta tecnología en el área de educación. Este tratadista hace referencia al caso de IBM, corporación que hace algunos años propuso el concepto de "gemelo digital cognitivo" aplicado a la manufactura. El objetivo del proyecto, fue y sigue siendo, enriquecer a un gemelo digital con capacidades cognitivas, tanto internamente como a través de la detección con capacidad cognitiva. La noción de que un gemelo digital podría ser una entidad cognitiva, por lo tanto, poseer conocimiento y 
ser capaz de explotarlo (una base de datos puede almacenar conocimiento, pero no puede explotarlo; no "sabe" que está almacenando conocimiento) es interesante. Esto último, da a entender el autor, es lo que, precisamente, puede representar en la educación una perspectiva de incalculable valor de investigación, estudio y desarrollo.

Leclère también ha sugerido que, la evolución de la tecnología de los GD ha sido rápida, refiriendo como ejemplo de ello el uso que están haciendo de ella las grandes empresas tales como: General Electrics, por medio de su plataforma loT para sus turbinas de viento, a fines de hallar una solución para protegerlas de los vientos violentos del Atlántico norte; Rolls Royce, quien usa la tecnología de GD para diseñar digitalmente diferentes escenarios con los que se puedan obtener mejoras para el diseño de sus motores de avión; y SAP; quien ha formulado un componente de la solución SAP Leonardo, Ilamado SAP Predictive Engineering Insights, el cual se sirve de un programa 3-D y lo combina con los datos enviados por los sensores, como la fuerza o el estrés, para dar una visión sobre los dispositivos. Este componente se integra con otros componentes como SAP Predictive Maintenance and Service, así como SAP Asset Intellingence Network.

Ciprés (2020) proporciona otro ejemplo de evolución al referir el uso de GD por parte de la reconocida empresa italiana Maserati (parte de la empresa matriz Fiat), quien ha logrado en la fabricación de dos de sus autos, una coexistencia integral entre los robots y operarios humanos, mediante completa digitalización del proceso productivo en base a la tecnología de los GD. El éxito de este proyecto fue posible en virtud la asociación con Siemens y el software desarrollado por ésta (CAD NX) con el que ha sido posible alcanzar beneficios tales como: el ahorro en costes, la reducción de errores, la mejora de la calidad de los automóviles, la identificación de posibles cue- llos de botella sobre los que trabajar, y el acortamiento tanto del time to market como del tiempo de desarrollo de nuevos vehículos, todo ello traduciéndose en una genuina ventaja competitiva.

\section{Utilidades y Ventajas de los GD}

Los ámbitos de aplicación más destacados para esta tecnología son: la aeronáutica, la automoción, industria, planeación de ciudades, mercadeo e incluso salud (medicina); y a su vez, más específicamente en áreas de concepción y diseño, mantenimiento preventivo, servicios y la relación con el cliente y formación (Leclère, 2018).

Adicionalmente se ha extraído que, con los GD también es posible:

- Comprobar si un producto cumple con las especificaciones ideadas en su diseño antes de hacer un prototipo real o de entrar en producción.

- Identificar si un producto es estable e intuitivo sin tener que incurrir en costosas pruebas de laboratorio o test a gran escala.

- Monitorizar posibles fallos en los productos en base a muchas más simulaciones que las que podríamos obtener en un entorno tradicional.

- Detectar ineficiencias o áreas de mejora en los procesos de fabricación de una planta industrial.

- Mejorar el mantenimiento preventivo de la maquinaria empleada y probar distintos modelos de producción sin tenerlos que implantar en la operativa diaria, reduciendo los riesgos asociados a estos cambios.

- Reducir el tiempo de inactividad de las fábricas y optimizar el consumo de energía.

- Crear un punto único de visualización de los datos procedentes de las fábricas, centralizando el Big Data de la empresa, reduciendo los costes de administración y proporcionando mejores variables a

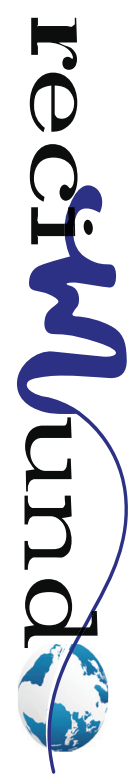


los directivos a la hora de gestionar el negocio.

- Agilizar el análisis exhaustivo de la información, aspecto éste que, al ser armonizado con procedimientos de Big Data, IoT e IA, facilita el control minucioso de los sistemas para evadir complicaciones, esquivar tiempos de parada, intentar nuevas oportunidades de negocio, concebir eventuales escenarios mediante simulaciones y especializar la producción a partir de las demandas de los clientes.

- Proveer una retroalimentación instantánea de la operación en curso, aplicando correctivos de manera más pronta, aspecto que viene siendo particularmente valioso para el mantenimiento de máquinas y equipos conectados que generan y analizan grandes volúmenes de datos.

- Detectar anticipadamente anomalías, incidencias o cualquier avería del propio funcionamiento interactuando a la par con los operadores humanos de manera directa.

- Favorecer el funcionamiento completamente autónomo, gracias a la capacidad que estos sistemas poseen en examinar escenarios, formular soluciones optimizadas y ponerlas en funcionamiento.

- Facilitar la corrección de errores y generan versiones nuevas y más confiables.

- Reducir costos, ya que, la evaluación de distintas soluciones respecto a los problemas que se plantean con un determinado producto, su entorno o proceso de producción, se consigue sobre el análisis en un entorno digital, sin necesidad de incurrir en inversiones de tiempo y dinero en pruebas con algo físico.

- Incrementar la productividad y mejora la eficiencia.

- Mejorar la calidad; gracias a los múltiples ensayos, simulaciones y predicciones que se pueden hacer con anticipación, y también; favorece la obtención de ventajas de mercado si, de forma directa, se brinda la oportunidad a los propios usuarios el acceso a soluciones de GD comprensibles. (BBVA, 2019; De Juana, 2020; Iberdrola, 2020; Mapfre Global Risks, 2020).

\section{Barreras Tecnológicas en el estableci- miento de los GD}

Aunque las ventajas asociadas a los GD son mayores que las desventajas, se hallaron coincidencias en los siguientes puntos clave que dificultan la materialización de su implementación, entre esas están:

- La dificultad técnica para digitalizar y monitorizar de manera intensiva los procesos en la industria, que cuenta con gran variedad de dispositivos, sistemas legados aislados, buses de campo, protocolos propietarios, así como una estricta arquitectura de integración y automatización industrial.

- La incapacidad de los sistemas actuales con que cuentas las industrias para el almacenamiento y tratamiento de los volúmenes de datos que se requieren en la invención y evolución de los GD, que sean verdaderamente representativos del desempeño de los elementos físicos, y no sólo sus particularidades y su estado.

- La complejidad tecnológica asociada a los requerimientos de las representaciones digitales de avanzada que conduzcan a escenarios de manejo de múltiples copias digitales simultáneas, con mayor capacidad de evaluar escenarios alternativos. (ITI, 2020)

\section{Conclusión}

Basados en las fuentes de datos revisadas para la presente investigación es posible atreverse a deducir que, a pesar de que la tecnología de los GD representa un avance relativamente reciente, los expertos aseguran que ha tenido una rápida evolución y aplicación en varios tipos de industria, sin embargo, debe tenerse presente que la generación de un GD debe asumirse como un 
proceso de creación de un sistema evolutivo en el transcurrir del tiempo, con un diseño que se va efectuando por etapas, de manera tal que en cada una de éstas pueda delimitarse sus costos y aceptables expectativas, siendo en base a la ejecución de dichas etapas el medio por el que puedan obtenerse beneficios y el desarrollo de las siguientes fases de construcción del propio GD.

También fue posible inducir que, la finalidad de crear un GD no es la conseguir la consumación de la entidad o réplica virtual en sí misma, sino más bien los beneficios que de éste es posible obtener.

En definitiva, es posible estimar que la evolución de los GD estará estrechamente vinculada a la propia evolución tecnológica y a la, cada vez mayor, admisión en distintas áreas e infraestructuras digitales en industrias, productos, ciudades y otras; mismas que favorecerán cada vez el acceso a software e integradores que facilitan nuevos emprendimientos de este tipo de proyectos tecnológicos con importes cada vez más factibles para las empresas.

\section{Bibliografía}

Alfonso, M. (03 de julio de 2018). Qué es un Digital Twin o gemelo digital y de dónde vienen? Recuperado el 30 de octubre de 2020, de https://www. economiadehoy.es/noticia/33238/tecnologia/quees-un-digital-twin-o-gemelo-digital-y-de-dondevienen.html

BBVA. (30 de enero de 2019). ¿Qué es un gemelo digital y para qué sirve? Recuperado el 30 de octubre de 2020, de https://www.bbva.com/es/ como-se-gana-el-hackathon-bbva-2020-los-vencedores-nos-lo-cuentan/

Cámara Valencia. (s.f.). Portal servicios, comercio y otros > Sectores> Agroalimentario. Recuperado el 30 de octubre de 2020, de https://ticnegocios. camaravalencia.com/servicios/tendencias/ayuda-gemelo-digital-digital-twin-al-desarrollo-negocio/

Ciprés, D. (01 de octubre de 2020). Gemelo Digital para industria y procesos logísticos. Recuperado el 30 de octubre de 2020, de https://www.itainno- va.es/blog/logistica/gemelo-digital-para-industria-y-procesos-logisticos/

De Juana, R. (13 de Julio de 2020). Gemelos digitales, aliados de la industria en el futuro. Recuperado el 30 de 10 de 2020, de https://www. muycomputerpro.com/2020/07/13/gemelos-digitales-aliados-de-la-industria-en-el-futuro

Geinfor. (s.f.). Gemelos Digitales en la Industria 4.0. Recuperado el 30 de octubre de 2020, de https:// geinfor.com/business/gemelos-digitales-en-la-industria-4-0/

Iberdrola. (2020). Gemelos digitales, claves en la Cuarta Revolución Industrial. Recuperado el 30 de 10 de 2020, de https://www.iberdrola.com/innovacion/gemelos-digitales

ITI. (30 de junio de 2020). Digital TwinsS: Gemelos Digitales en la transición a la Industria 4.0. Recuperado el 30 de octubre de 2020, de https://www. iti.es/proyectosidi/proyecto-gemelos-digitales-industria-4-0/

Leclère, R. (octubre de 2018). El auge del "gemelo digital" gracias al IoT en la Industria 4.0. (A. d. España, Ed.) Ausape(57), 30-31. Recuperado el 30 de octubre de 2020, de https://ausape.com/ documentos/Media/Publicaciones/Revistas/2018/ R57_Ausape.pdf

Mapfre Global Risks. (18 de febrero de 2020). melos Digitales, el camino hacia la eficiencia industrial. Recuperado el 30 de octubre de 2020, de https:// www.mapfreglobalrisks.com/gerencia-riesgos-seguros/articulos/gemelos-digitales-el-camino-hacia-la-eficiencia-industrial/

Rodríguez, A. (11 de junio de 2019). Hablemos de gemelos digitales. Recuperado el 30 de octubre de 2020, de https://news.sap.com/latinameri$\mathrm{ca} / 2019 / 06 /$ hablemos-de-gemelos-digitales-bl0g/\#: :text=Los\%20\%E2\% 80\%9Cdigital\%20 twins\%E2\%80\%9D\%200\%20gemelos,que\%20 no\%20ten\%C3\%ADa\%20conexi\%C3\%B3n\%20 f\%C3\%ADsica.\&text=Son\%20la\%20evoluci\%C3\%B3n\%20de\%20aquellos,los\%20entornos\% 


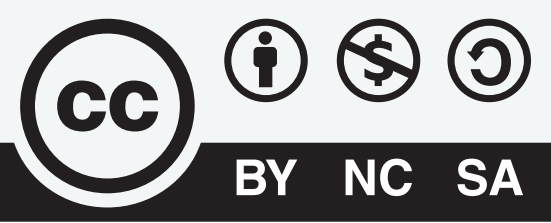

CREATIVE COMMONS RECONOCIMIENTO-NOCOMERCIAL-COMPARTIRIGUAL 4.0.

\section{CITAR ESTE ARTICULO:}

Varas Chiquito, M., García Plua, J. C., Bustamante Chong, M., \& Bustamante Chong, C. (2020). Gemelos digitales y su evolución en la industria. REClMUNDO, 4(4), 300-308. https://doi.org/10.26820/recimundo/4.(4).noviembre.2020.300-308 